\title{
Optically pumped mirrorless lasing. A review. Part I. Random lasing
}

\author{
Nastishin Yu. A. and Dudok T. H. \\ Institute of Physical Optics, 23 Dragomanov Street, 79005 Lviv, Ukraine
}

Received: 27.06 .2013

\begin{abstract}
Currently optically pumped mirrorless lasing is represented by three distinct branches, which concern lasing in different types of the gain media: optically random and photonic media, and microcavities. This article is a first part of our review on optically pumped mirrorless lasing, with the random lasing in scattering media being a main subject. The other mirrorless lasing mechanisms will be addressed in the second part. Considering light localization as a key function of the feedback, we discuss possible mechanisms for the light localization in the scattering media. Special attention is paid to the Anderson light localization. The other mechanisms of the light localization in the scattering media concern high Q-resonances in local microresonators, which exist due to structural inhomogeneities in the scattered media. Applications of the random lasers are shortly reviewed.
\end{abstract}

Keywords: mirrorless lasing, mirrorless lasers, random lasing, random lasers, light localization, optical feedback, amplified spontaneous emission, superfluorescence, superrradiance

PACS: 42.55.Zz; 42.55.Sa; 42.55.Tv; 42.60.Da; 42.55.Mv; 42.70.Hj; 42.25.Dd/ UDC: $535.37+535.35+681.7 .069 .24+52-626$

\section{Introduction}

Nowadays there are not too many people in the civilized world who have never seen a laser light or, at least, never heard about it. A vast majority of people certainly associate lasing (or laser generation) with a light source called LASER. Less of them, probably mostly specialists in optics, readily decipher the term LASER as an acronym for Light Amplification by Stimulated Emission of Radiation. And still less, probably mostly professionals in laser physics are aware that the latter phrase of five words is not enough for the appropriate definition of laser generation. An essentially important issue called optical feedback is missed in this word construction. The notion of the optical feedback concerns the type of light amplification, at which the emitted light is returned back along the same path (which topologically is equivalent to a closed loop) for amplifying light over and over again. A presence of feedback is a conditio sine qua non for true lasing. With no feedback a corresponding light amplifying source would reduce to an optical amplifier only.

Feedbackless light amplification can be based on one of the following phenomena: amplified spontaneous emission (ASE), superradiance (SR) or superfluorescence (SF). In this review lasing is defined as a feedback-amplified stimulated light emission under an action called pumping. Strictly speaking, the presence of optical feedback implies coherent light amplification. However, to tell the truth, some of the authors use the term 'lasing' in its historically original sense defined by the acronym LASER as alluded to above, which (rather unintentionally) covers also the case of incoherent amplification of spontaneous emission and is termed in the current literature as lasing with nonresonant (incoherent) feedback. Under the feedback action, newly emitted and multiply returned light waves interfere in a feedback resonator. The resonator is either an external optical 
cavity built of mirrors, bounding a gain medium or a gain medium per se. In the latter case one says that the feedback is mirrorless. Two remarks are in order here.

The first remark concerns the role of interference of the light waves at the optical feedback. If the interfering light waves are coherent, then due to the constructive and destructive interference a standing light wave is formed. De facto the formation of the standing wave implies that the light wave is localized within the resonator cavity. However de jure the term light localization is reserved for another, more specific, phenomenon, which concerns the state of light wave in a disordered medium and which is a light analogue of the phenomenon of Anderson localization of electrons in disordered solids. Both the formation of the standing wave in the cavity of an optical resonator (further called cavity light localization) and the Anderson light localization refer to a regime, at which the light wave is essentially trapped in space and thus it would be reasonable to cover these two cases by the common term light localization. The Anderson light localization is also called strong localization to distinguish it from yet another regime called weak localization in a disordered medium. Further in the text we use the term light localization as a general category, which covers different specific light localization mechanisms such as cavity light localization, strong (or Anderson) light localization, and weak light localization. The light localization is a key function of the optical feedback and its action is twofold: first, it essentially traps the emitted light within the gain medium for avalanche-like enhancing the stimulated emission and, second, it sets up selection rules for lasing lines. The lasing lines are resonance modes of the emitted light, which survive under interference. The selection rules for the lasing modes are determined by conditions of the constructive interference at which the phase difference between the interfering waves is a multiple of $2 \pi$. The phase difference between the interfering waves is governed by the geometrical and optical parameters of the resonator and/or the gain medium. In conventional lasers the feedback is achieved with specially assembled optical schemes of mirrors. The simplest and the most popular Fabry-Perot laser resonator is built of two facing mirrors [1], though more complicated schemes are also designed, depending on the desired laser properties.

The second remark concerns the notion of mirrorless lasing. Mirrorless lasing can be implemented if the optical gain is combined with multiple light scattering in random media, selective Bragg reflection in photonic crystals, total internal reflection in closed microcavities or surface plasmons in metallic nanoparticles. As a result, the multiple light scattering is a mechanism of optical amplification for so called random lasers. The selective light reflection represents a mechanism that provides optical feedback in naturally occurring photonic crystals such as cholesteric and blue-phase liquid crystals, as well as in artificially fabricated photonic structures. The total internal light reflection in closed cavities in the shapes of solid spheroids or liquids and liquid crystal droplets leads to so called 'whispering gallery resonance modes' in microlasers. Finally, the surface plasmon resonances provide the optical feedback in spasers, nanoplasmonic counterparts of the lasers.

Currently there are three mirrorless laser technology branches: random lasers, photoniccrystal lasers and microcavity lasers develop independently. It is logic to foresee that soon they should interpenetrate each other. There are at least following arguments for this. Progress in all the three areas is dictated by common practical needs in multifunctional portable devices based on mirrorless lasers. To achieve the goal, recent there have appeared the ideas to combine together different mirrorless mechanisms in a single laser. This is indeed possible since there are numerous examples [2-9] when different mirrorless mechanisms take place simultaneously (see Section 3 for details). In spite of these points, still there has been no review considering all the three mecha- 
nisms together. A fortiori, the current literature (see the recent reviews on the random lasers [10, $11]$, the liquid crystal lasers [12], and the microlasers [13, 14]) contains almost no cross-references among the articles belonging to these different branches of mirrorless laser technology.

The aim of this work is to draw together literature information on the mirrorless feedback mechanisms for optically pumped lasing in different media. In this article which is a first part of our review, the random lasing in the scattering media is considered. A second part devoted to lasing in the photonic crystals and the microcavities will appear in the forthcoming issue of this journal.

\section{Random lasers}

Random lasing is the light amplification due to stimulated emission in a multiply scattering gain medium. The term random lasing was coined by Wiersma and co-workers $[15,16]$. Although this type of lasing has attracted attention of researchers only recently, it has been explored soon after invention of the traditional lasers with coherent feedback. It has been shown $[17,18]$ that the laserlike emission exists even if one of the mirrors is replaced by a scattering element. This means that a light-scattering gain medium can provide efficient amplification even with no external mirrors. This idea has been used for the explanation of laser-like emission by the interstellar medium. The next subsection is a brief sketch on the development of a paradigm of cosmic lasers.

\subsection{Paradigm of a cosmic microwave laser}

It is believed [11] that the first lasing has been observed nearly a century before the first optical laser is made. According to Ref. [11], which refers to the website [19], these are stars discovered by Wolf and Rayet in 1867. Although it might be possible, we do not find enough arguments to share this opinion and follow a more commonly accepted version, according to which an interstellar microwave laser has been discovered in 1960s due to studies of molecular emission spectra of $\mathrm{OH}$-radicals from the interstellar medium [20]. Hundreds of microwave cosmic lasers based on the $\mathrm{OH}$ emission have been discovered since then. Other chemical species $\left(\mathrm{CH}, \mathrm{H}_{2} \mathrm{CO}, \mathrm{H}_{2} \mathrm{O},{ }^{15} \mathrm{NH}_{3}\right.$, $\mathrm{CH}_{3} \mathrm{OH}, \mathrm{SiS}, \mathrm{HC}_{3} \mathrm{~N}, \mathrm{SiO},{ }^{29} \mathrm{SiO},{ }^{30} \mathrm{SiO}, \mathrm{H}$ etc.) present in the interstellar space have been qualified as such that show some features of the amplified spontaneous emission. For more details on the astrophysical lasers, the reader is encouraged to read Refs. [20-22].

Below we summarize the main results collected by astronomers in 1960s, which allow claiming the existence of the cosmic microwave laser. These are as follows: (1) anomalous intensity ratios for the $\mathrm{OH}$ emission lines leading to the temperatures up to $2 \times 10^{6} \mathrm{~K}$, (2) narrow spectral width of the emission lines, (3) their strong polarization for the isotropic character of absorption, (4) narrow beaming of the emission, and (5) time variations of the intensities.

Such highly intriguing results have attracted attention of physicists working in the field of laser generation. The amplified spontaneous emission in a cosmic gain medium has been considered theoretically by Varshalovich [23]. Soon Letokhov [24] has demonstrated theoretically that scattering on free electrons or interstellar dust can provide efficient amplification in the interstellar medium. Such a cosmic microwave laser supposes to share features of its laboratory-built prototype of conventional laser with the mirror-based feedback, namely a threshold for pumping energy, spectrally narrow emission lines, a strong polarization, a narrow beaming and a high brightness, though it should reveal low coherency and time variability, or even pulse-like character of the emitted radiation. The feedback in such lasers is nonresonant.

It is worthwhile to compare the cosmic microwave laser with its laboratory-built counterparts. First, similarly to the traditional laboratory-built lasers, the cosmic microwave laser is based 
on the inverted population of energy states, but the way the inverted population is created is different; no pumping in its usual sense is involved. Three main excitation mechanisms have been suggested [22]: collisional excitation, excitation by recombination, and a Bowen mechanism [25, 26]. Second, strictly speaking, the cosmic laser represents an optical amplifier rather than a laser by the physical principle of its operation. An optical amplifier differs from the true laser by the absence of optical feedback. This issue will be analyzed in more details in Subsection 2.3. Third, the cosmic laser works in the continuous-wave (CW) regime, whereas the pulse regime is easier in its realization for the laboratory-built lasers. And fourth, the cosmic laser emits in the $4 \pi$ solid angle, whereas the light of the traditional lasers is essentially directional.

\subsection{Laboratory-built lasers with nonresonant feedback}

To achieve lasing with the nonresonant feedback, the authors of Ref. [17] have replaced one of the mirrors in the laser resonator by a volume scatter or a surface scattering plate. Since in such a laser the feedback is not resonant, the lasing modes do not depend on the path length of the beam within the optical cavity. The main lasing frequency corresponds to the resonant frequency of the gain medium. The narrowing of the emission line results from the increasing number of photons at the energy level corresponding to the frequency of the maximum gain.

The innovative importance of the lasing scheme where one of the mirrors of the resonator is replaced by a scattering element $[17,18,27]$ is twofold. First, it has raised a question of possibility of lasing with incoherent feedback. Second, it has become clear that the laser-like amplification can be produced without a mirror-based resonator - spatial inhomogeneity of the refractive index in the gain medium can provide the conditions for the increase of the light path in the medium. It has opened a way for development of a wide variety of lasers with nonresonant feedback.

It is understood that the theory of random lasing should be based on the theory of light scattering. The light scattering theory is very complicated in general. Simplified description can be obtained in different approximations. In the frame of Rayleigh scattering approach the scatterers are considered as spheres of the diameter much smaller than the wavelength of light. A Mie theory deals with the scattering of spherical particles of any diameter, i.e. it does not have a lower limit for the size of the scattering centres. It reduces to the Rayleigh scattering when the diameter of the particles is much smaller than the light wavelength, such that in this limit the shape of the particles no longer matters. However, even with these approximations the scattering theory is still quite complicated and further simplifications are used. One of the approximations concerns the concentration of the scattering particles. At low and high limits for the concentration of scatterers, the scattering regimes are called weak and strong scattering. The key parameters distinguishing between these regimes are the free path length $\Lambda$, on which the direction of the propagation of a photon is randomized, the size of sample $L$, the wavelength of light $\lambda$, and the diameter $D$ of the scattering particle. In the most practical cases, the size of the sample is much larger than the size of the scattering centres $(L \gg D)$ and also larger than the wavelength $(L \gg \lambda)$. Then the scattering regime is governed by the relation between $\Lambda$ and $L$. At small concentrations of the scatterers, the scattering is weak and one accepts that $\Lambda \gg L$. At higher concentrations the scattering is strong such that the opposite inequality $L \gg \Lambda \gg \lambda$ is valid. The weak scattering approximation $(\Lambda \gg L)$ applies, for instance, to the scattering in the interstellar medium [24].

Extension of the light scattering theory to the gain media for the description of the random lasing additionally introduces two important length: the gain length $\Lambda_{g}$ and the loss length $\Lambda_{l}$. The 
gain (loss) length is the length for which the intensity of light waves travelling inside the gain medium increases (decreases) $e$ times. The lasing threshold is defined by the condition $\Lambda_{g}=\Lambda_{l}$ [28].

Although the model of diffusion scattering in a gain medium developed by Letokhov [29, 30] is a great simplification that neglects the wave nature of light, it is very powerful in its predictions. For example, it shows that there exists a threshold at which the radiation losses are compensated by the gain emission and above which the lasing starts. As a consequence of this energy threshold, a critical condition for the size of lasing sample arises. It turns out that if the diffusion approximation is satisfied $(L \gg \Lambda \gg \lambda)$, then a general relation $L_{c r}=\sqrt{\Lambda \Lambda_{g} / 3}$ holds true, independent of the sample shape.

The consideration of dynamics shows that the time dependence of the lasing intensity above the threshold is has a shape of pulsations, with decaying amplitude. After the pulsation regime the system reaches a stationary lasing. Such pulsations are typical transition processes of lasers. In the stationary state, the dynamics of the laser emission is akin to the black body radiation and so isotropic. The spectral density of the emitted radiation also displays temporal dynamics in the form of spectral narrowing of the laser line. This natural property of the lasing with nonresonant feedback has been studied for the first time in Ref. [31]. For quite a while line narrowing (both spectral and temporal) and transient intensity pulsations have been considered as a sort of markers distinguishing the true lasing from the ASE, until Diederik Wiersma and Ad Lagendijk [32] have demonstrated numerically that these features are also properties inherent of the ASE, which works with no feedback.

The Letokhov's theory [29, 30] also confirms the experimental evidences that the radiation from the laser with nonresonant feedback consists of a large number $\left(N \sim 10^{9}\right)$ of strongly coupled modes (i.e., waves propagating in different directions).

Though the theory of lasing with nonresonant feedback based on the approach of diffuse light scattering in a gain medium has premised the development of the conception of mirrorless lasing, it does not represent a generic idea behind this conception. Probably, for this reason there has been no significant progress in the area for more than fifteen following years. The research activities have been focused on the development of lasers with mirror-based stochastic and chaotic resonators. A breakthrough has been done by John [33, 34] who introduced conception of localization of photons in disordered media, in analogy with the Anderson localization of electrons in disordered solids. John has shown that ordinary non-confined dielectrics in which the light propagation is described in the diffusion approximation (when the multiple scattering interference effects are neglected) do not localize light, but the light localization might appear if the interference effects of multiple scattering are taken into account. The light localization is sufficient and crucial for the presence of optical feedback in the mirrorless lasers. Below we briefly discuss the importance of the light localization for realizing optical feedback in the mirrorless lasing.

\subsection{Light localization and optical feedback}

In the most general case, the feedback implies that the two or more processes are connected in a loop such that each process influences the other [35]. In application to the physics of signals, a positive feedback would mean that a signal is generated inside a closed loop, along which it is multiply amplified. If the signal is multiply attenuated within the loop instead, then one says that the feedback is negative. In the simplest case the loop is formed between the input and output of an amplifier (see Fig. 1a). 


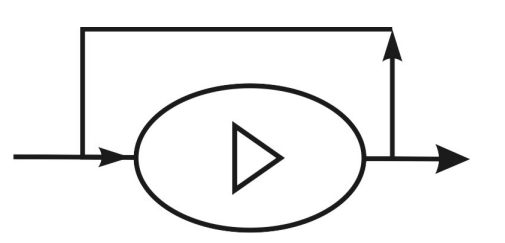

(a)

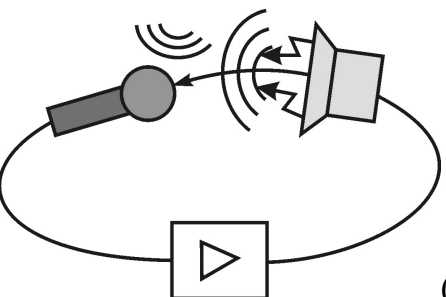

(b)

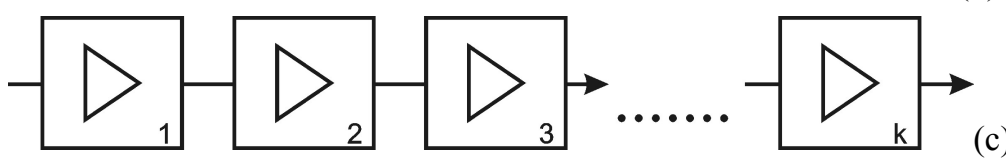

Fig.1. Scematic representation (a) of feedback amplification, (b) audio feedback, and (c) feedbackless amplification.

An example illustrating a feedback loop is the audio feedback (Fig. 1b), at which a sound signal is transformed into an electrical signal by a microphone, then amplified, reproduced as a sound by a loudspeaker and then again sent to the same microphone, the same amplifier, the same speaker, and so on along the loop. One can say that when the positive feedback is present, the signal is essentially localized in the feedback loop. It has to be noticed that the presence of the amplifier in the scheme shown in Fig. $1 \mathrm{~b}$ already implies an inherent feedback loop inside the amplifier. This internal feedback loop is necessary for the amplifier to function as such. However it is not this loop that provides the audio feedback. A closed loop providing the audio feedback is shown in Fig. $1 \mathrm{~b}$ by the solid line connecting the microphone, the amplifier, the loudspeaker and the same microphone. A presence of the closed loop is a distinctive feature of the feedback. For this reason we consider further the amplification along an open loop as a feedbackless process [35] in the same vein as the open loop is, in fact, not a loop at all.

A cascade-like amplification at which several amplifiers are connected in a series, as in Fig. 1c, is considered as a feedbackless amplification scheme, too. A two-mirror resonator in traditional lasers is an example of an optical feedback. Due to the bounding mirrors, the light signal travelling forth and back between the mirrors is localized within the cavity containing a gain medium. The closed feedback loop is geometrically formed by the forth-and-back trajectories of light within the resonator cavity. As a difference with an electronic feedback scheme, where the inputoutput direction is not equivalent to its reverse (output-input), the forth and back directions in an optical gain medium are equivalent, thus forming a loop topologically. While applied to the optically pumped light emission, the two processes that might be connected into a loop are the light emission and amplification. The amplified spontaneous emission is an example of amplification along an open loop. Under diffusive light scattering, the trajectory of a photon is that of a random walk and the probability of its return to the same point is very small [36]. Even if the photon trajectory self-crosses, it does not form a loop because the photons do not scatter at each other. A loop trajectory could be formed only if photons return again to the same scatter. Such a situation would correspond to the light localization. If the photon is scattered along an open trajectory, sooner or later, after many zigzags, each photon leaves the medium. As a result, the total path length of the light inside the scattering gain medium is greatly increased though there is no feedback loop in this process; the light is trapped for a while within the scattering gain medium but is not localized. The laser generation in the absence of light localization reduces to one of the feedbackless emission mechanisms such as the ASE, the SR or the SF.

John $[34,37]$ has argued that the localization of light in a strongly scattering medium might take place in a light wavelength gap between the regions of extended states corresponding to the 
Rayleigh scattering and the spectral region corresponding to the light propagation described by geometrical optics. This issue will be explained in more details in the next subsection. Here the term 'extended state' simply means that a light wave propagates through a sample and finally exits from it, whereas the term 'localized state' means that photons are trapped within the sample and can leave it only due to optical leakage, through an imperfect resonator.

The light localization in disordered optical media as a phenomenon has been introduced in analogy with the Anderson localization of electrons in disordered solids. For the benefit of a reader we briefly consider this analogy in Subsection 2.4.

\subsection{Scattering-mediated localization of light and random lasing}

The Anderson effect of electron localization in disordered solids [38] is rooted in the wave nature of matter which shows up at the microscopic scales. Electrons in disordered solids are subject to interaction with spatially aperiodic electromagnetic field of the solid lattice. For electrons moving along the coordinate $x$ in a spatially random potential $V(x)$, the quantum states are described by the Schrődinger equation

$$
\left[-\frac{\hbar^{2}}{2 m^{*}} \nabla^{2}+V(x)\right] \psi(x)=W \psi(x) .
$$

Electrons with sufficiently negative energy $W$ may get trapped (localized) in the regions where $V(x)$ is deep enough. Only electrons whose energy is greater than some critical value $W_{c}$ escape the energy traps and traverse through the solid, thereby conducting electricity, contrary to the electrons with the energies lower than $W_{c}$, which do not contribute to electric conductivity. Such a phenomenon of trapping of electrons in disordered materials is called the Anderson localization, named after Philip Anderson who has discovered the phenomenon theoretically in 1958. As a result of the Anderson localization, a metal might become an insulator at sufficient degree of disorder. The critical value $W_{c}$ is called the mobility edge; the term has been coined by Nevill Mott, who has developed greatly the theory of electron localization [39]. The Anderson localization is strongly sensitive to the degree of disorder, which can be characterized by the mean free path $\Lambda_{e}$ of electrons in a solid. The localization takes place when $\Lambda_{e}$ becomes comparable to the De Broglie wavelength $\lambda_{D}$ of the electron. $\Lambda_{e}$ decreases with increasing degree of disorder at a weak disorder. Since the rate for tunnelling the electron out of the deep potentials depends on the probability to find nearby the potential fluctuations into which the trapped electron can tunnel at further increase of disorder, the value of $\Lambda_{e}$ starts to increase due to easier tunnelling of electrons at closer packing of the potential wells. In terms of energy, the latter means that the mobility edge moves into the conduction band $(W>0)$. That is why there are two mobility edges and the localized states form a band gap on the energy diagram.

Naturally, a question of whether photons can be localized in a strongly scattering medium has been raised. A quick glance to the problem considered in the diffusion approximation (when the multiple scattering interference effects are neglected) leads to the answer that ordinary nonconfined dielectrics do not localize light. As shown by John [34], this can be seen comparing the Schrödinger equation (see Eq. (1)) to the wave equation for the electric field of a light wave with the frequency $\omega$ and the speed $c$ propagating in a medium with spatially inhomogeneous dielectric constant $\varepsilon=\varepsilon_{0}+\varepsilon_{\text {fluct }}(x)$ : 


$$
\vec{\nabla}^{2} \vec{E}+\vec{\nabla}(\vec{\nabla} \cdot \vec{E})-\left(\frac{\omega}{c}\right)^{2} \varepsilon_{\text {fluct }}(x) \vec{E}=\left(\frac{\omega}{c}\right)^{2} \varepsilon_{0} \vec{E}
$$

In what follows below in this paragraph, we present the arguments for the analogy between Eq. (1) and Eq. (2) given by John in Ref. [34]. A comparison of Eq. (2) and Eq. (1) shows that $\vec{E}$ in Eq. (1) plays a role of the wave function $\psi$ in Eq. (1); the term $(\omega / c)^{2} \varepsilon_{\text {fluct }}(x)$ responsible for scattering of the light wave in Eq. (2) corresponds to the random potential $V(x)$ in Eq. (1); the term $(\omega / c)^{2} \varepsilon_{0}$ is an analogue of the eigenvalue $W$ in the Schrödinger equation (see Eq. (1)); finally, the term $\vec{\nabla}(\vec{\nabla} \cdot \vec{E})$ has no analogue in Eq. (1). This term is of crucial importance when the light interference effects at multiple scatterings are taken into account, but is usually neglected in the diffusion approximation, when one assumes that a plane wave propagating in a disordered medium loses the information on its initial polarization on the scales much larger than the photon free path $\Lambda$.

In the diffusion approximation the vector nature of the photon field can be disregarded, such that $\vec{E}$ reduces to its amplitude $E(x)$ and Eq. (2) becomes similar to a scalar equation given by Eq. (1). However, this is similar only to some extent, because there is also a difference between the equations. The difference is that the energy $W$ of an electron in the medium can be sufficiently negative and thus the electron may be trapped in the regions where $V(x)$ is negative and deep, whereas in the case of electromagnetic light wave the term $(\omega / c)^{2} \varepsilon_{0}$ (which is the analogue of the eigenvalue $W$ in Eq. (1)) is always positive. As a result, the light wave with the positive eigenvalue $(\omega / c)^{2} \varepsilon_{0}$ cannot be localized in the negative deep $-(\omega / c)^{2} \varepsilon_{\text {fluct }}(x)$. Moreover, contrary to the electronic system, where the localization can be achieved with increasing $\lambda_{D}$ (lowering the energy of an electron), in case of light the increase of the wavelength $\lambda$ (lowering the energy of a photon) is accompanied by a tendency to weakening the scattering. Indeed, the term $-(\omega / c)^{2} \varepsilon_{\text {fluct }}(x)$ in Eq. (2), which is the analogue of the random potential $V(x)$ in Eq. (1)) and responsible for scattering, vanishes at $\omega \rightarrow 0(\lambda \rightarrow \infty)$. The opposite high-frequency limit corresponds to the regime of geometric ray optics. Basing on the above arguments, John [33,34] has concluded that, in contrast to the electronic disordered systems, the light cannot be localized in diffusively scattering and fully disordered medium. This qualitative conclusion is confirmed by more precise recent considerations [40] accounting the vector nature of light.

It is intuitively understood that the light wave will not propagate through a scattering medium if the photon free path length $\Lambda$ is not longer than the light wavelength $\lambda$. Hence, the condition $(\Lambda / \lambda)>1$ specifies the extended states for a strongly scattering medium, whereas $(\Lambda / \lambda)<1$ defines the localized light states. This can be understood considering a light wave described by its electric field $E=E_{0} e^{-i(\omega t+k z)}$, where $\omega$ is the frequency and $k=2 \pi / \lambda$ the wave number. The wave is not formed (mathematically it means that $E$ is not a periodic function) if $k z<2 \pi$, which is equivalent to $\Lambda<\lambda$. Generally $\Lambda$ is a function of $\lambda$. There is no analytical expression $\Lambda(\lambda)$ valid for any $\lambda$, even for a dispersion of spherical scatterers in an isotropic medium. However it is 
known that $\Lambda(\lambda)$ is not a monotonous function (see Fig. 2). In the Raleigh scattering regime corresponding to the region of long wavelengths, one has $\Lambda(\lambda) \sim \lambda^{4}$ and, consequently, we have $(\Lambda(\lambda) / \lambda) \sim \lambda^{3} . \Lambda$ decreases with decreasing $\lambda$ in the Rayleigh scattering regime, but only to certain extent; $\Lambda$ is never smaller than the correlation length $\xi$ of the scatterer in the given host medium. To explain the physical meaning of $\xi$, let us recall that for a single sphere of the radius $R$ the scattering cross-section is equal to $2 \pi R^{2}$, which means that the sphere 'feels' the light wave on the distance $\xi=\sqrt{2} R$ from the centre of the sphere, which is larger than $R$. For a dense packing of spheres, the $\xi$ value is a characteristic parameter governed by their radii, their optical contrast, and the concentration of spherical scatterers (i.e., the inter-particle distance). For short wavelengths $(\lambda<\xi)$. the length $\Lambda(\lambda)$ increases with decreasing $\lambda$, in agreement with expectations of the geometrical optics. Therefore $\Lambda(\lambda)$ is a nonmonotonous function, which has a minimum at $\Lambda(\lambda)=\xi$. In the case of large-size scatterers $(R \gg \lambda)$ and their dilute dispersion, the $\xi$ value is larger than $\lambda$ for the whole spectrum and the inequality $\Lambda(\lambda) / \lambda<1$ has no solution (see Fig. 2a). For dense packing of the spheres, when the size of the scatterers and the inter-particle distance are comparable to $\lambda, \xi$ might become smaller than $\lambda$ (see Fig. 2b). However, because the correlation length $\xi$ is significantly larger than the sphere radius, after reaching the condition $\Lambda=\xi$ and further increase in the concentration of scatterers, the scattering cross sections of the neighbour spheres overlap. One says that the spheres become 'optically connected' and because of this $\Lambda$ starts to increase with increasing filling factor. Overlapping of the sphere-scattering cross sections resembles overlapping of the potential deeps outside the second mobility edge, which leads to the extended states in the electronic disordered systems, as alluded to above. These arguments indicate that the condition $\xi<\lambda$ for the localization of light is hard to achieve but, as remarked by John [34], it can be realized as a result of quite delicate interplay of different factors, in particular the size of the scatterers, their optical contrast and the filling factor in the presence of short-range order in their spatial packing.
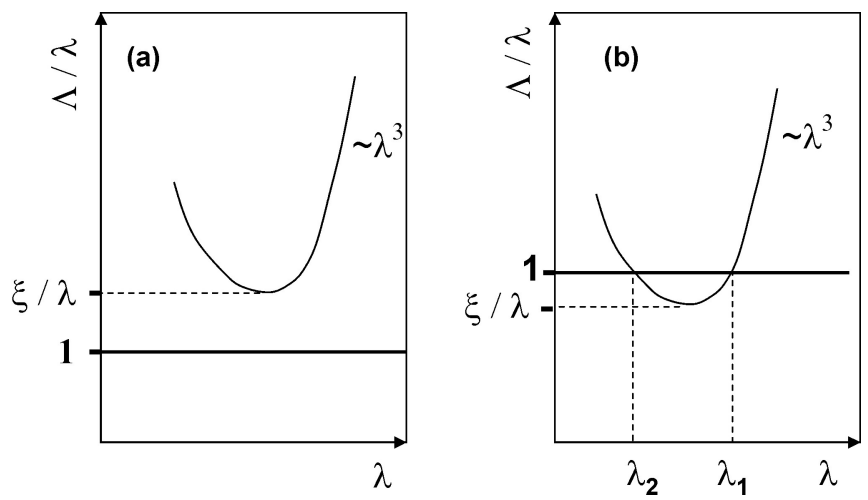

Fig. 2. According to Ref. [34], $\Lambda / \lambda$ vs. $\lambda$ is a nonmonotonous function for a scattering medium. The inequality $\Lambda / \xi<1$ has no solution for large-size scatterers (a), for which $R \gg \lambda$ and thus $\xi>\lambda$ for any $\lambda$; it is valid for densely packed small-size scatterers (b), for which $\xi<\lambda$ in the wavelength gap between the regions of extended states corresponding to the Rayleigh scattering and the spectral region corresponding to light propagation described by the geometrical optics. 
The notion of weak light localization is worth discussing. The phenomenon of weak localization of electrons has been discovered by four authors [41] in 1979. A simple approach to the problem of weak electron localization in real space has been developed by Khmelnitskii [42]. Afterwards this approach has been adopted to the problem of strong light localization (see Ref. [43] and references herein). The idea of this approach is as follows. For a system of closely packed spherical scatterers of the radius $R$ and the inter-particle distance $d$ such that $\lambda \gg R \simeq d$, the light wavelength $\lambda$ covers a domain embracing the sizes of many particles and thereby the determination of the photon trajectory is not an evident procedure. To set it on a quantitative basis, one calculates the probability $W$ for the amplitudes $A_{i}$ as $W=\sum\left|A_{i}\right|^{2}$. Notice that, besides the purely squared terms, here one deals also with the cross-terms $A_{i} A_{j}^{*}$ accounting for the interference effects, which are absent when the form $W=\Sigma\left|A_{i}^{2}\right|$ is used. There is always a nonzero probability that the photon trajectory is a closed loop and, because of the symmetry arguments, the two alternative directions along the loop have equal probabilities. In such a case due to the constructive interference between the two waves circulating along a loop in the opposite directions, the light is strongly scattered backward. This is the phenomenon of weak light localization. Therefore, the weak light localization is a result of the interference effects in the scattering medium, at which the scattering is strictly backwards.

The authors of Ref. [43] argue that once the loop is formed, it stimulates the formation of another loop and so on. This is a kind of a feedback. As a result, photons become trapped in the system of loops. A standing wave is formed within a spatial region of the size $\sim \lambda$. This is nothing else but the phenomenon of light localization in a scattering medium. According to Ref. [43], the cases of the weak and strong (Anderson) light localizations correspond to two topologically different sets of photon loops. Under weak localization, the photon trajectories form a set of separate degenerated (with respect to the travelling direction) loops, whereas under strong localization, the loops develop on an arbitrary photon trajectories. However this is not a whole picture for the strong localization. The photon elastic scattering length $\Lambda_{s}$ must be smaller than the photon absorption length and yet $\Lambda_{s}$ must be of the order of $\lambda$. There will be no light localization at $\Lambda_{s} \gg \lambda$. Under appropriate conditions, both the weak and strong light localizations can serve as feedback mechanisms for the lasing in the scattering gain media.

Invention of the conception of light localization has led to other new conceptions such as photonic crystals and photonic amorphous structures, thus leading to the conception of mirrorless lasing in photonic gain media. Introduction of intriguingly sounding terms 'semiconductors of light', 'random laser' and 'photonic lasing crystal' has attracted great attention and stimulated increase in the research activities. Great expectations and revolutionary predictions have been announced in photonics $[34,44,45]$. This optimism has been supported by some beliefs that the light localization in the photonic scattering media will not suffer from the difficulties caused by satellite phenomena of the type of those met for the electron localization in the disordered solids. In this respect, John has remarked that the optical systems provide an ideal experimental realization of a single non-interacting excitation in a static random potential [37] since, in contrast to electrons, their transport is not affected by the Coulomb interaction in the most practical cases beyond the quantum electrodynamics and so one accepts that photons do not scatter at each other [46-48]. However it has turned out soon that the analogy between the electron and light localizations shares similar difficulties in their experimental identification. The electron localization that leads to low- 
ering electron mobility and is expected to show up as a significant increase in the electrical resistance, is essentially masked by the electron-electron interaction and electron-phonon scattering in any real materials [49]. Similarly, the Anderson light localization in the scattering media is masked by accompanying optical phenomena and an unequivocal proof of the existence of light localization in the disordered bulk materials is still lacking. A serious obstacle in these studies is absence of a criterion handy for experimental identification of the Anderson light localization in a random gain medium.

The light localization is an easy matter for the media with lower dimensions $(D<3)$ since, according to the scaling theory of localization, which applies to both electrons in disordered solids and electromagnetic waves in disordered dielectrics, all the states are localized in 1D and 2D media [34, 41, 50-52]. However, theoretical consideration and experimental detection of the light localization in 3D samples is still a challenging problem [34].

The light localization can be detected while studying coherency of the backscattered light, though the difficulty is that the backscattered light can be partially coherent due to weak localization even for a fully random medium [53-56]. The other possibilities for detection of the light localization in randomly scattering media have been suggested in Refs. [46, 48, 57]. From the theoretical point of view, the situation is also complicated by the conclusion recently made by Skipetrov and Sokolov [40]: fully disordered systems are not suitable for observation of the Anderson localization of light and the very possibility of this phenomenon for the light is doubtful for any 3D media without sufficient structural correlations.

The first suggestion for probing the light localization is due to John [33] who has shown that the presence of the photon edge mobility can be detected as an anomalous increase in the light absorption, when the critical frequency is approached. Soon Anderson [58] has proposed that the reflectivity of a randomly scattering slab (which, according to his remark, can be considered as an effective absorption) should decrease near the photon mobility edge. These two possibilities are in line with the theoretical predictions and the experimental observations that the light emission by a gain medium (mutatis mutandis, a negative light absorption) shows up as lasing at the edge of the photonic band gap.

The coherent lasing at the photon mobility edge could be an optical mark of the light localization. The difficulty is that the resonant coherent laser emission is not always easily identified against its nonresonant counterpart, especially in the case when different lasing modes overlap. In addition, the lasing regime is hardly discriminated versus the alternative amplification mechanisms, which are based on single-photon path and thus do not require any feedback (namely, the SR [59], the SF [60-62] and the ASE). Similarly to the laser emission, the SR and the SF are also coherent emissions, whereas the ASE can also be coherent under some conditions. To be on the same page with a reader, below we will recall in short the physical sense of these phenomena.

Generation of photons resulting from the relaxation of electrons from their excited state to the ground state is called spontaneous emission. The phases of the emitted photons are random in the case of the spontaneous emission. One says that the emitted light is not coherent. As a consequence, the total energy of $n$ incoherent photons is equal to $n A^{2}$. Were photons synchronized in their phases (a coherent light), the total energy would be equal to $n^{2} A^{2}$. Einstein has predicted that, when the excited states are highly inversely populated, then a photon emitted previously can stimulate the emission of another photon, which appears to be in phase with the stimulating photon. In their turn, the two newly emitted photons can further stimulate the emission of other photons 
and so on. This is a so called stimulated emission. The stimulated emission is also called as a negative absorption. Since the excited inversely populated states are highly unstable, the energy accumulated from the pumping source can be released back through the stimulated emission in a relatively short time. Shortening of the emission time leads to increasing power of the emitted light. The light energy accumulated from the pumping source is released back by a short pulse. When the excited states are highly inversely populated and the path length for the photons in the gain medium is long enough under condition of high enough pumping energy, the number of the emitted photons shows avalanche multiplication and the spectrum of the emitted light significantly narrows, becoming centred at the frequency (wavelength) corresponding to the maximum gain. This is the ASE, also called superluminescence. The ASE is thresholdless in nonscattering media but, as discussed below, it might manifest a well defined threshold in strongly scattering gain media.

There are two other light emission amplification mechanisms called $S R$ and $S F$. The SR is a cooperative emission of an ensemble of atoms (or ions) coupled by a pumping pulse into a coherently excited collective state owing to a macroscopic dipole moment. Thus it is emitted cooperatively. Then the number of the emitted photons increases in a kind of chain reaction. Due to the coherence of the emission, the intensity emitted by $n$ atoms is proportional to $n^{2}$, instead of $n$ as for the incoherent spontaneous emission [63]. The phenomenon of SR has been predicted by Dicke in 1954 [59]. If, at low pumping intensities, the emission starts as incoherent uncorrelated process without a dipole moment induced by the pumping field and then, at higher pumping, the exited atoms become coupled by their own common radiation field, giving rise to an induced macroscopic dipole, and thereby becoming synchronized and cooperatively emitting the stored energy, then the emission is called SF [64]. Since the emitted light is coherent in the case of SF, the emitted intensity is also proportional to the squared number of the emitting atoms $\left(n^{2}\right)$. The SF is the cooperative emission of fluorescent light by the excited atoms without the dipole moment below the pumping threshold and with the latter dipole moment in their common radiation field above the threshold [60, 61, 65-67]. At the same time, the SR represents a similar process, though the macroscopic dipole leading to the coherent emission is nonzero even below the threshold. Both the SR and the SF appear as pulses of high intensity with temporally and spectrally narrow profiles. In contrast to the ASE, which is a collective incoherent process, the SR and the SF are coherent cooperative emissions. In the literature, many authors do not distinguish among the phenomena of $\mathrm{SF}$, SR and superluminescence (which is another name for the ASE). The SR, SF and the ASE can compete in certain situations and a transition from the SF to the ASE has been reported in Ref. [68].

One of the features frequently used for discriminating between the ASE and the laser generation is a threshold character of the latter. However, the ASE in the randomly scattering media [69] and, in some cases, in the nonscattering media [53, 54, 70-73] exhibits a threshold behaviour as well. The thresholds in these cases correspond to the pumping energy, at which the light gain overcomes the light losses. The SF and the SR are also threshold processes [60-62] and, thus, additional efforts are needed to exclude them from consideration. Nonetheless, when reporting a laser-like emission, often these feedbackless amplification mechanisms are simply not discussed.

An optical analogue of a so called Thouless number $\delta=(\delta E) /(d E / d N)$ [74], which has been introduced for the electron localization in disordered solids, could be a light-localization criterion. Here $\delta E$ is a measure of broadening of an energy level $E$ due to a finite lifetime of electrons on that level and $d E / d N$ the spacing of the energy levels. In application to the localization 
of light, we have $\delta=(\delta v) /(d v / d N)$, where $\delta v$ and $d v / d N$ are respectively the frequency width and the spacing of the excited states [75]. Experimentally the $\delta$ parameter may be determined as a ratio of the full width at half maximum (FWHM) denoted by $\delta v$ for the light emission line to the spectral spacing $\Delta v$ between the neighbouring emission lines [36]. The extended (nonlocalized) states correspond to $\delta>1$, which implies that the modes spectrally overlap, and the localization of light is expected at $\delta<1$, which corresponds to well separated spectral modes. Evidently, one has $\delta<1$ for the conventional lasing with the mirror-based feedback, since the FWHM for the laser modes is of the order of $0.1 \mathrm{~nm}$ and different laser modes are single or well separated spectrally. For the lasers with nonresonant feedback the inequality $\delta \gg 1$ holds true, since a large number $\left(N \sim 10^{9}\right)$ of the coupled modes are overlapped (implying zero inter-mode spacing). This forms a lasing band, which can still be quite narrow (about several nanometres). Chabanov et al. [48] have argued that the Thouless number cannot serve as a universal localization parameter in the presence of absorption, because its exponential scaling with sample size and its small values $(\delta<1)$ can be due to the absorption. Even if the laser-like lines are well separated, a special care must be taken to prove that these emission lines are not due to the ASE, SF or the SR.

On one hand, the maximum of the ASE line is expected at the wavelength of the maximum gain but. On the other hand, the results [76] has testified that it can be shifted with respect to the position of the fluorescence maximum in nonscattering media due to many different factors related to the shapes of the absorption and scattering spectra, the geometry of the excitation beam, the size of the sample, etc. Therefore the spectral position of the emission line cannot be used as a signature discriminating between the ASE and the true lasing.

Frolov et al. [77] have reported the emission spectra with the features as narrow as $0.1 \mathrm{~nm}$ in various scattering gain media, including thin $\pi$-conjugated polymer films, organic dye-doped gel films, and opal crystals saturated with polymer and laser dye solutions. The authors [77] argue that the ASE model fails to explain the finely structured emission spectra in the saturation intensity range. They assign these emission lines to the laser-like emission regime.

The mechanism of diffusive scattering, which is a basis for the concept of nonresonant feedback, implies increase in the total light path length but does not rely on the return of a photon to a previously visited point. The photon path in a diffusively scattering medium is that of a random walk, and the probability of a photon trajectory returning upon itself is small [66] for the diffusive media with the Thouless number $\delta \gg 1$, which is the very case for the lasing with nonresonant feedback. Apalkov, Raikh and Shapiro [78] have argued that even if such loops could exist the light amplification along them is unrealistic, because some portion of light is free to scatter out of the loops, which should result in a very high lasing threshold. In Refs. $[77,79,80]$ it is argued that there might be nonclosed trajectories in strongly scattering media, with extremely long total light paths along which the light is amplified. Whether this is true or not, the original picture suggested by Lethokhov et al. [17, 29, 30] implies the very open, loopless photon trajectory. A loopless character of the photon trajectory raises a doubt about applicability of the concept of feedback, which by its definition is the amplification along a closed loop (see Subsection 2.3). The very open character of the photon trajectory in the diffusively scattering medium corresponds to a cascadelike amplification scheme, which is termed as the ASE for the optical gain media.

Therefore, one is led to conclude that the light emission above the pumping energy threshold in the diffusively scattering gain media de facto is feedbackless emission governed by the ASE, the $S F$ or the $S R$, though since the time of its discovery de jure it is termed as a lasing with nonreso- 
nant feedback. In the current literature the nonresonant (incoherent) laser feedback is also called intensity or energy feedback and the resonant (coherent) laser feedback is also called field or amplitude feedback [81]. It is in spite of the emission with the intensity or energy feedback is equivalent to that peculiar for the nonresonant (incoherent) feedback, which actually is feedbackless. The terms 'lasing (or laser) with the intensity/energy/nonresonant/incoherent feedback' are widely used in the current literature and so, when using this term or citing the other authors, one has to keep in mind that the emission amplification in these cases is actually feedbackless.

As shown by Letokhov [30], the total gain in the scattering gain medium is proportional to its volume, whereas the light losses are proportional to the square of the surface of the excited sample. Then the emission intensity starts to be amplified if the linear size of the sample is larger than a critical value $L_{c r}$. The presence of $L_{c r}$ is an inherent property of the ASE in the scattering medium: the light path must be long enough to overcome the light losses. It is this reason that the ASE has been observed for the pumping beams of a stripe-like shapes but not for the circular cross sections $(0.5-1 \mathrm{~mm}$ in diameter) [82-87].

The strength of the scattering can be controlled through the concentration, size, shape and the refractive index of scatterers. If the light is scattered by the polymer-dispersed liquid crystal droplets, then the refractive index and so the scattering strength can be controlled by the voltage applied. Ref. [6] has reported the 'random lasing' in a dye-doped polymer-dispersed liquid crystal. The observed field-controlled anisotropy of the emission has been explained in terms of the scattering-mediated feedback switched from a 3D random walk to a quasi-2D type. In our opinion, this explanation does not account an important detail: the scatterers are spherical containers, which might play a role of resonant cavities producing so called whispering gallery lasing modes. Since the whispering gallery modes are equatorial modes, the optical pumping along the cell normal (e.g., a $Z$ direction) should yield in the maximum lasing parallel to the cell plane ( $X-Y$ plane), thus agreeing with the observations reported in Ref. [6].

The threshold character of the optically pumped emitted intensity, together with the spectral and temporal narrowing of the emission line have been reported for different scattering media such as crystal powders [2, 3, 88, 89], dispersions of solid-particle dye solutions [76, 90-93], organic films and opal crystals [77].

The difficulties in identification of the light emission regime about the energy threshold in the random media addressed above, have led to some terminology ambiguities. Terminological arguing would be of no importance if this led to no difficulties in understanding the physics of the underlying processes. A representative illustration of such difficulties is debates between the research groups by Wiersma [94] and Lavandy [95] concerning whether the optically pumped light emission in the strongly scattered media observed by Lavandy et al. [96] is the true lasing or the ASE. Lavandy and co-workers [95] have qualified the light emission occurred in dispersion of nanoparticles in a dye solution as 'laser action', while Wiersma and co-workers [94] have argued that the results of Lavandy et al. [96] have to be explained in terms of the ASE.

The experiments for the light emission in the powders of luminescent microcrystals have brought some evidence for the features which point to the coherent resonant feedback. Unusual observations of the light emission lines akin to the resonant modes of the conventional lasers have been reported in Refs. $[2,3,77,87,88]$ for the powder microcrystals. The threshold character of the emission intensity enhancement and the spectral and temporal narrowing of the emission lines are their common features. The spectral shape of the emission has been found to be dependent on the size and shape of microcrystals. Only one emission line located at the maximum of the lumi- 
nescence band has been observed in the powders of microcrystals with the isometric shape, thus suggesting a feedbackless emission amplification mechanism, whereas for the powders of microcrystals with clearly anisometric shape several narrow $(0.1 \mathrm{~nm})$ emission lines have been detected [97]. The number of the lines is larger for larger pumping intensities and excitation areas. These features suggest that the microcrystals might play a role of microcavities providing optical feedback. Then the coexistence of both feedbackless and feedback mechanisms of the emission amplification and interplay between them should be common for the microcrystalline powders.

The observations by Cao et al. [93, 98-102] of highly intriguing spectrally narrow emission peaks (called 'spikes') in the microcrystalline powders and the polycrystalline films of $\mathrm{ZnO}$ have raised intense debates on the origin of these spikes. Cao et al. [93, 98-106] qualify these spikes as being due to lasing with the coherent feedback provided by multiple scattering effects. This opinion has been shared and further developed by Sebah and Vanneste [107] and Apalkov et al. [78]. Alternatively, Mujumdar et al. [79] and Pinheiro and Sampaio [80] have suggested possibilities for explaining these spikes by the ASE along extremely long light paths, which might be rare but extremely effective for the light emission.

To understand the nature of the emission in $\mathrm{ZnO}$ powders, the statistics of the light emission has been studied [102]. The experiments show that, when the pumping intensity increases, the photon emission statistic changes from the Bose-Einstein type, which is expected for the singlemode chaotic light, to the Poissonian one, which should be characteristic for a single-mode coherent emission. However, the Poissonian distribution is expected not only for the single-mode coherent light requiring optical feedback, but also for the multimode chaotic emission, which can be feedbackless. This fact introduces ambiguity into the answer to the question of whether the emission with the Poissonian statistics is a true lasing.

To explore these problems better, Cao et al. [100] have studied the transition between the two emission amplification regimes, which have been assigned to the ASE and the lasing, while varying the concentration of $\mathrm{ZnO}$ nanoparticles dispersed in the methanol solution of Rhodamine 640 percholate dye. The dependence of the emission intensity as a function of pumping intensity reveals two thresholds at high enough $\mathrm{ZnO}$ concentrations $\left(\sim 5 \times 10^{11} \mathrm{~cm}^{-3}\right)$. The narrowing of the emission line is accompanied by strong enhancement of the emission intensity. These observations are similar to those reported by Lawandy et al. $[96,108]$ and are explained as the ASE, which represents a feedbackless mechanism, in spite of being qualified as the lasing with nonresonant feedback in the current literature. The emission band collapses (see Fig. 3a) from $15 \mathrm{~nm}$ (spectrum 1) to $5 \mathrm{~nm}$ (spectrum 2) in its spectral width with achieving the first threshold, thus signalling about the ASE regime. The second threshold corresponds to the appearance of narrow $(0.2 \mathrm{~nm})$ peaks against the background of the ASE spectral line (see Fig. 3b). These peaks have been qualified as the lasing with coherent feedback. When the particle concentration increases up to $\sim 10^{12} \mathrm{~cm}^{-3}$, the discrete narrow peaks are appearing prior the collapse of the emission band (see Fig. 3c), against the background of which they stand at lower concentrations. This fact has been explained as evidence that the threshold for the emission regime attributed to the laser generation with coherent feedback appears to be lower than the threshold for the ASE.

In Ref. [87] the laser-like emission from the nanocrystalline powders has been explained as a collective emission from an ensemble of crystalline grains, with a kind of distributed feedback provided by multiple light scattering.

While studying the distribution of spatial extent of the modes in a porous gallium phosphide filled with a methanol solution of rhodamine 640 perchlorate acting as a random laser, Molen et al. [109] have documented an interplay between essentially single-mode and distinctly multimode behaviours. It has been shown that almost 80 per cent of the modes have a spatial extent smaller 
than the diameter of the pump-light spot size $(2 \mu \mathrm{m})$. This latter shows that the random laser ynder study is in a crossover regime where the localized modes prevail. A theory attempting to explain discreetness of the emission spectrum for the feedbackless diffusive random lasers [110-112] has been developed by Türeci et al. and applied [112] to interpret the results obtained in Ref. [109]. The laser theory is formulated in terms of bi-orthogonal modes called as constant-flux states. Strong interaction in the multimode diffusive random laser leads to the effect termed as a 'spatial hole-burning' [113]. According to the idea, the modes that lase first tend to suppress lasing in the other modes, which they are correlated with in space. As a result of this effect, the surviving lasing modes appear to be 'thin' and well separated spectrally.

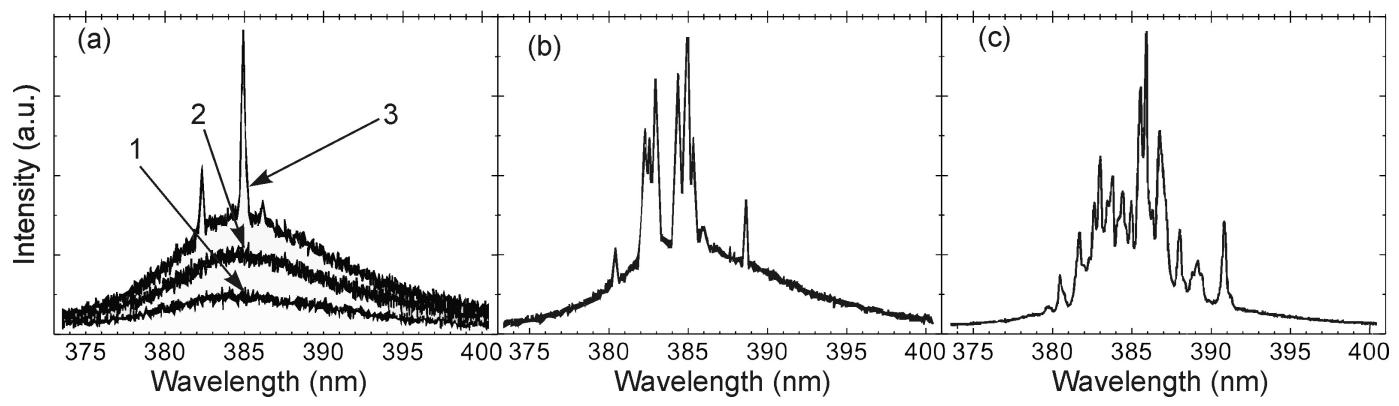

Fig. 3. Schematic representation of evolution of the emission spectra for $\mathrm{ZnO}$ nanoparticles dispersed in the methanol solution of Rhodamine 640 percholate dye for different pumping intensities, as observed in Ref. [100]: (a) emission spectrum for moderate $\left(\sim 5 \times 10^{11} \mathrm{~cm}^{-3}\right) \mathrm{ZnO}$ concentrations below (spectrum 1) and above (spectrum 2) the first (ASE) threshold, and above the second (lasing) threshold (spectrum 3); (b) the same observed well above the second threshold; (c) illustration of the fact that the lasing threshold gets lower than that for the ASE for higher $\mathrm{ZnO}$ concentrations $\left(\sim 10^{12} \mathrm{~cm}^{-3}\right)$.

Ref. [88] has been claimed to be a first observation of lasing in the regime of strong Anderson localization of light. The mean size of microcrystals has been of the order of several tens of nanometres, i.e. much smaller than the light wavelength. The inter-particle spacing has been less than $\lambda / 15$. The transport length of photon before complete randomization (a free path length of photon) has been measured to be less than half the wavelength (namely, $114 \mathrm{~nm}$ at $\lambda=363.8 \mathrm{~nm}$ and $311 \mathrm{~nm}$ at $\lambda=632.8 \mathrm{~nm}$ ). It is worth recalling that in the conditions when the closed path providing feedback for the lasing is shorter than half the wavelength, the constructive and destructive interference of light loose their conventional sense, precluding spatial directionality of lasing or mode selectivity and leading to independence of the intensity on the observation angle, just as expected for truly 'random' laser action. It is interesting that a mode-locking transition has been observed in randomly scattering gain medium under test (micrometre-sized clusters of titania nanoparticles with static disorder, immersed in a rhodamine dye solution clusters) [114].

Finally, it is worth mentioning that the mirrorless lasing technology experiences explosive development at present, as a consequence of discoveries in different though joined by the same goal areas. In addition to well established mirrorless lasing mechanisms of the random lasing in the scattering media, a topic of lasing in photonic media and microcavities has recently emerged in nanoplasmonics, being an intriguingly interesting possibility for the mirrorless lasing. A counterpart of the mirrorless lasers has been suggested by Bergman and Stockman [115] basing on surface plasmons (SPs) in noble metal (gold, platinum, silver etc.) or aluminium and alkaline metal nanoparticles. It is called SPASER (see $[116,117]$ among the recent review), which is an acronym for Surface Plasmon Amplification by Stimulated Emission of Radiation. 
The first experimental demonstration of a spaser-based nanolaser is due to Noginov et al. [118]. Unlike the cases discussed above where the internal feedback might be present under special conditions, the feedback represents an inherent property for the surface plasmonic nanopaticles, which cannot be removed. The SP resonances in the metal nanoparticles are strongly localized oscillating electron excitations. Strong localization of the SPs is the origin of their inherent feedback. However, there is also a serious obstacle because of strong light absorption in metals. The authors of Ref. [118] have advised compensating the light loss by the optical gain and demonstrated that the spaser can emit coherent light if the nanoparticles are adjacent to the gain medium. Nevertheless, it is important to stress that a spaser as such is not a laser. It is rather a resonator that provides localization of the oscillating electric fields and leads an inherently present feedback. A laser can be built out of a spaser when combined with a gain material. When the gain molecules are adjacent to the spaser and excited with a pump beam, they do not emit photons but transfer the energy to the resonant SPs via non-radiative transitions. In their turn, the SPs further stimulate non-radiative transitions that transfer more energy to other SPs and so on, thus accumulating more and more SPs which are strongly coherent. There is a one-to-one correspondence with the stimulated emission of photons. The SPs in a spaser play a part of photons in a laser. The SPs accumulate in a spaser until the threshold is reached and then they might start to emit photons. A spaser can also be considered as a generator of local electric fields. It turns out that the electromagnetic fields in the optical range are almost purely electric oscillations on the nanoscales, whereas the magnetic field component is small and does not contribute to the optical effects [119]. An interesting feature of such a generator is that it generates both 'luminous' and 'dark' optical fields. The 'dark mode' is a SP, which does not emit a photon, but it can transform into a luminous mode if, for example, the symmetry of a spaser nanoparticle is broken; then the spaser becomes a laser. Zheludev et al. [120] have suggested using a metamaterial containing a planar array of spasers with slightly perturbed symmetry. They have demonstrated that such a construction constitute quite efficient planar laser that emits light perpendicular to its plane.

\subsection{Applications of random lasers}

The prospects of applications of the random lasers, both coherent and incoherent, are related to their advantages when compared with the conventional mirror-based lasers. The main advantage of the random laser is that it is simple in fabrication and cheap in production. It can be prepared in the shape of a tablet or liquid suspension, which can be used as it is or being painted on a surface of, say, a road pavement, vehicles, ships, aircrafts, or as lighting on secure dressing. Incoherent random lasing has been suggested for using in speckle-free imaging [121]. Randomness of the emission characteristics of the random lasers can be employed for generation of random numbers [122, 123]. It is known that a computer cannot generate absolutely random numbers. Instead it produces 'pseudorandom' numbers, following special algorithms. In this respect one can quote a famous expression by John von Neumann who has once said "Anyone who considers arithmetical methods of producing random digits is, of course, in a state of sin". To overcome this problem, one can use naturally occurring phenomena with a random character of produced signals. The random lasing is one of the promising options for this purpose.

If a random suspension of nanoparticles capable for the random lasing is printed onto a document, then such a printed mark can serve as an authentication sign of the document [124]. Decoding the document can be performed by its irradiation with pumping light and a detector is used to analyze the spectrum of emerging laser light. Supernarrow peaks of the coherent random lasers are due to high Q-resonances in microcavities, which have random geometrical parameters and thus 
and thus are unique for each sample. A random character of the resonant frequencies produced by these microcavities enables authenticity of the 'laser label' attached to a document [125]. The random lasing could also be used while detecting chemical impurities in liquids, including water, or in novel displays with extremely high switching speeds and resolutions. It is also supposed that the random lasing technique can be used for detecting injuries in human tissues [126]. In this case a tissue doped with a laser dye serves as a scattering gain medium, and its internal cellular structure determines spectral features of the lased light. A tissue sample containing a tumour will lase differently compared to a healthy tissue. However, this possibility, along with many others, still have to be verified and implemented in practice.

\section{Concluding remarks}

This article constitutes a first part of the review on optically pumped mirrorless lasing. It is devoted to optically pumped random lasing in the scattering gain media. The second part, to appear in the next issue of this journal, will consider the optically pumped mirrorless lasing in photonic media and microcavities.

The idea of mirrorless lasing takes its origin from the concept of lasing with nonresonant feedback occurring in diffusively scattering media. The same idea has already led to the paradigm and discovery of cosmic lasers. A presence of optical feedback is a conditio sine qua non for lasing. In the absence of optical feedback, a light-amplifying source represents a light amplifier rather than a laser. Because of open character of photon trajectories in diffusively scattering media, the lasing with the nonresonant (incoherent) feedback in such gain media becomes de facto a feedbackless light amplification. For this very reason, the cosmic laser addressed above is a light amplifier, too. The term 'random lasing' has been introduced intentionally for the lasing with the resonant (coherent) feedback.

In what the phenomenon of resonant feedback mediated by strong scattering in a gain medium concerns, the highly challenging John's idea of the Anderson light localization still remains the most attractive and frequently studied. In spite of this, the question of whether the lasing with the feedback due to the Anderson localization of light can ever be convincingly observed in strongly scattering optical gain media still remains open. Both practical implementation and identification of this lasing mechanism are difficult and, because of this, no clear evidence for the optical feedback based on the scattering-mediated strong localization of light has so far been presented, except for maybe Ref. [88].

Sharp spectral emission peaks reported up to date for the randomly scattering gain media have been associated with high Q-resonances, though their origins appear to be not universal. These may be the total internal reflection in microscrystals serving as microcavities, or the Mie or Bragg resonances in the photonic glasses composed of dye doped microspheres [4]. The other examples of the appropriate media are 2D amorphous nanostructures of air holes embedded in a high-index semiconductor [127], artificially fabricated amorphous photonic structures [7] that mimic isotropic nanostructures with local short-range order and produce noniridiscent colours in nature $[128,129]$, quasiperiodic photonic crystal structures $[8,9]$ utilizing localized optical resonances, aperiodic structures of pseudorandom morphologies [130], and quasicrystalline structures with the Fibonacci sequence in 1D grating geometry of the conventional distributed-feedback laser [131]. It would be also of great interest to check the possibilities of a so called transverse localization of light [132] for laser generation.

Though a tendency to develop different branches of mirrorless lasers independently is still lasting, it would be reasonable to foresee that soon they will interpenetrate. The arguments for this 
are as follows. The progress in all of the three areas is dictated by common practical needs in devices based on mirrorless lasers, which are technologically simple in fabrication, cheap in production, handy in use, multifunctional and portable. To achieve these goals, recently there have appeared the ideas to combine together different mirrorless mechanisms, yielding in a single laser. This is indeed possible since there have been numerous examples when different mirrorless mechanisms take place simultaneously. The examples are diverse and numerous: the random lasing in microcrystalline powders $[2,3]$ where the microcrystals play a role of microcavities; the resonance-driven random lasing in disordered self-assemblies of monodisperse microspheres, which show the Mie resonances [4]; the lasing in dye-doped liquid crystal droplets, with different mechanisms of optical feedback such as the Bragg selective reflection in cholesteric droplets [5] or the random lasing [6] in polymer dispersed liquid crystal droplets (for both of them whispering gallery modes can be simultaneously expected because of spherical shape of the liquid crystal droplets); the lasing in amorphous photonic structures where the random long-range positional ordering coexists with the local crystalline one [7], or with the long-range orientational ordering in artificially fabricated quasicrystals $[8,9]$.

The random lasers represent one of at least three distinct branches in the mirrorless laser technology. The two other branches are associated with lasing in the photonic crystals and the microcavities. The reader is invited to follow the review of the latter branches in the following issue of this journal.

\section{Acknowledgement}

This work is supported by the Grant 0113 U000468 from the Ministry of Education and Science of Ukraine.

\section{References}

1. Milonni P W and Eberly J H. Laser physics (New Jersey: John Wiley \& Sons Inc. Hoboken, 2010).

2. Fork R L and Taylor D W, 1979. Unusual optical emission from microcrystals containing $\mathrm{Eu}^{+2}$ : Experiment. Phys. Rev. B. 19: 3365-3400.

3. Markushev V M, Zolin V F and Briskina Ch M, 1986. Luminescence and stimulated emission of neodymium in sodium lanthanum molybdate powders. Sov. J. Quant. Electron. 16 (2): 281-283.

4. Gottardo S, Sapienza R, Garsia P D, Blanco A, Wiersma D S and Lopez C, 2008. Resonancedriven random lasing. Nature Photonics. 5: 429-432.

5. Humar M and Musevic I, 2010. 3D microlasers from self-assembled cholesteric liquid-crystal mi- crodroplets. Opt. Express. 18: 26995-27003.

6. Gottardo S, Cavalieri S, Yaroshchuk O and Wiersma D S, 2004. Quasi-two-dimensional diffusive random laser action. Phys. Rev. Lett. 93: 263901-4.

7. Jin C, Meng X, Cheng B, Li Zh and Zhang D, 2001. Photonic gap in amorphous photonic materials. Phys. Rev. B. 63: 195107-5.

8. Notomi M, Suzuki H, Tamamura T and Edagawa K, 2004. Lasing action due to the twodimensional quasiperiodicity of photonic quasicrystals with a penrose lattice. Phys. Rev. Lett. 92: $123906-4$.

9. Nozaki K and Baba T, 2004. Quasiperiodic photonic crystal microcavity lasers. Appl. Phys. Lett. 84: 4875-4877.

10. Wiersma D S, 2008. The physics and applications of random lasers. Nature Phys. 4: 359-367.

11. Ramachndran H, 2002. Mirrorless lasers. Pramana - J. Phys. 58: 313-323. 
12. Coles H and Morris S, 2010. Liquid-crystal lasers. Nature Photonics. 4: 676-685.

13. Matsko A B, Savchenkov A A, Strekalov D, Ilchenko V S and Maleki L, 2005. Review of applications of whispering-gallery mode resonators in photonics and nonlinear optics. IPN Progress Rep. 42-162: 1-51.

14. Chiasera A, Dumeige Ya, F'eron P, Ferrari M, Jestin Y, Conti G N, Pelli S, Soria S and Righini G C, 2010. Spherical whispering-gallery-mode microresonators. Laser Photon. Rev. 4: $457-482$.

15. Wiersma D S, van Albada, M P and Lagendijk Ad, 1995. Random laser? Nature. 373: 203204.

16. Wiersma D S and Lagendijk Ad, 1996. Light diffusion with gain and random lasers. Phys. Rev. E. 54: 4256-4265.

17. Ambartsumyan R V, Basov N G, Kryukov P G and Letokhov V S, 1966. Laser with nonresonant feedback. JETP Lett. 3: 167-169.

18. Ambartsumyan R V, Basov N G, Kryukov P G and Letokhov V S, 1966. A laser with a nonresonant feedback. IEEE J. Quant. Electron. 2: 442-446.

19. The site http://home.achilles.net/ talbot/amateur/WolfRayet.html to which the author of Ref. [11] refers, seems to move to the address http://laserstars.org/amateur/WolfRayet.html

20. Letokhov V S, 2002. Lasing in space. Sov. Phys.: Uspekhi. 45: 1306-1309.

21. Johansson $S$ and Letokhov V S, 2002. Laser action in a gas condensation in the vicinity of a hot star. JETP Lett. 75: 591-594 (see also the website http://laserstars.org/news/EtaCarinae.html)

22. Letokhov V. S and Johansson S. Astrophysical lasers (New York: Oxford University Press, 2009).

23. Varshalovich D A, 1966. Coherent amplification of radio emission in a cosmic medium. JETP Lett. 4: 124-125.

24. Letokhov V S, 1966. Stimulated radio emission of the interstellar medium. JETP Lett. 4: 321323.

25. Bowen I S, 1934. The excitation of the permitted OIII nebular lines. Publ. Astronom. Soc. of the Pacific. 46: 146-150.

26. Bowen I S, 1935. The spectrum and composition of the gaseous nebulae. Astrophys. J. 81: 116.

27. Ambartsumyan R V, Kryukov P G, Letokhov V S and Matveev Yu A, 1967. Emission statistics of a laser with nonresonant feedback. JETP Lett. 5: 312-314.

28. Zaitsev O and Deych L, 2010. Recent developments in the theory of multimode random lasers. J. Opt. 12: 024001-14.

29. Letokhov V S, 1967. Stimulated emission of scattering particles with negative absorption. JETP Lett. 5: 212-215.

30. Letokhov V S, 1968. Generation of light by a scattering medium with negative resonance absorption. JETP. 26: 835-840.

31. Ambartsumyan R V, Kryukov P G and Letokhov V S, 1967. Dynamics of emission line narrowing for a laser with nonresonant feedback. JETP. 24: 1129-1134.

32. Wiersma D and Lagendijk Ad, 1996. Light diffusion with gain and random lasers. Phys. Rev. E. 54: 4256-4265.

33. John S, 1984. Electromagnetic absorption in a disordered medium near a photon mobility edge. Phys. Rev. Lett. 53: 2169-2172.

34. John S, 1991. Localization of light. Phys. Today. 44: 32-40.

35. Leigh J R, Control theory, $2^{\text {nd }}$ ed. (London: The Institution of Electrical Engineers, 2004).

36. Milner V and Genack A Z, 2005. Photon localization laser: Low-threshold lasing in a random

Ukr. J. Phys. Opt. 2013, Volume 14, Issue 3 
amplifying layered medium via wave localization. Phys. Rev. Lett. 94: 073901-4.

37. John S, 1987. Strong localization of photons in certain disordered dielectric superlattices. Phys. Rev. Lett. 58: 2486-2489.

38. Anderson P W, 1958. Absence of diffusion in certain random lattices. Phys. Rev. 109: 14921505.

39. Mott N F, 1967. Electrons in disordered structures. Adv. Phys. 16: 49-144.

40. Skipetrov S E and Sokolov I M, Absence of Anderson localization of light in a random ensemble of point scatterers. ArXiv:1303.4655v1 [physics.optics] 19 Mar 2013.

41. Abrahams E, Anderson P W, Licciardello D C and Ramakrishnan T V, 1979. Scaling theory of localization: Absence of quantum diffusion in two dimensions. Phys. Rev. Lett. 42: 673676.

42. Khmel'nitskii D E, 1984. Localization and coherent scattering of electrons. Physica B. 126: 239-241.

43. Maksimenko V V, Krikunov V A and Lushnikov A A, 1992. Strong localization of light in a closely packed granular medium. JETP. 75: 848-856.

44. Yablonovitch E, 1987. Inhibited spontaneous emission in solid-state physics and electronics. Phys. Rev. Lett. 58: 2059-2062.

45. Yablonovitch E, 2001. Photonic crystals: semiconductors of light. Sci. American. 285: 47-55.

46. Störzer M, Gross P, Aegerter Ch. M and Maret G, 2006. Observation of the critical regime near Anderson localization of light. Phys. Rev. Lett. 96: 063904-4.

47. Segev M, Silberberg Ya and Christodoulides D N, 2013. Anderson localization of light. Nature Photonics. 7: 197-204.

48. Chabanov A A, Stoytchev M and Genack A Z, 2000. Statistical signatures of photon localization. Nature. 404: 850-853.

49. Wölfe P and Vollhardty D, Self-consistent theory of Anderson localization: general formalism and applications, In '50 years of Anderson localization', Ed. by E. Abrahams (Singapore: World Scientific Publishing Co. Pte. Ltd, 2010).

50. John S, Sompolinsky H and Stephen M. J, 1983. Localization in a disordered elastic medium near two dimensions. Phys. Rev. B. 27: 5592-5603.

51. John S and Stephen M J, 1983. Wave propagation and localization in a long-range correlated random potential. Phys. Rev. B. 28: 6358-6368.

52. Azbel M Ya and Soven P, 1983. Transmission resonances and the localization length in onedimensional disordered systems. Phys. Rev. B. 27: 831-835.

53. Zyuzin $\mathrm{A} \mathrm{Yu}, 1994$. Weak localization in backscattering from an amplifying medium. Europhys. Lett. 26 (7): 517-520.

54. Wiersma D S, van Albada M P and Lagendijk Ad, 1995. Coherent backscattering of light from amplifying random media. Phys. Rev. Lett. 75: 1739-1742.

55. Van Albada M P and Lagendijk Ad, 1985. Observation of weak localization of light in a random medium. Phys. Rev. Lett. 55: 2692-2695.

56. Wolf P-E and Maret G, 1985. Weak localization and coherent backscattering of photons in disordered media. Phys. Rev. Lett. 55: 2696-2699.

57. Wiersma D S, Bartolini P, Lagendijk A and Righini R, 1997. Localization of light in a disordered medium. Nature. 390: 671-673.

58. Anderson P W, 1985. The question of classical localization. A theory of white paint? Phil. Mag. B. 52: 505-509.

59. Dicke R H, 1954. Coherence in spontaneous radiation processes. Phys. Rev. 93: 99-110.

60. Bonifacio R and Lugiato L A, 1975. Cooperative radiation processes in two-level systems: 
superfluorescence. Phys. Rev. A. 11: 1507-1521.

61. Arecchi F T and Courtens E, 1970. Cooperative phenomena in resonant electromagnetic propagation. Phys. Rev. A. 2: 1730-1737.

62. MacGillivray J C and Feld M S, 1976. Theory of superradiance in an extended, optically thick medium. Phys. Rev. A. 14: 1169-1189.

63. Scully M O and Svidzinsky A A, 2009. The super of superradiance. Science. 325: 1510-1511.

64. Maki J J, Malcuit M S, Raymer M G and Boydl R W, 1989. Influence of collisional dephasing processes on superfluorescence. Phys. Rev. A. 40: 5135-5142.

65. Rehler N E and Eberly J H, 1971. Superradiance. Phys. Rev. A. 3: 1735-1751.

66. Bonifacio R and Lugiato L. A, 1975. Cooperative radiation processes in two-level systems: superfluorescence. II. Phys. Rev. A. 12: 587-598.

67. Bonifacio R, Schwendimann P and Haake F, 1971. Quantum statistical theory of superradiance. I. Phys. Rev. A. 4: 302-313.

68. Malcuit M S, Maki J J, Simkin D J and Boyd R W, 1987. Transition from superfluorescence to amplified spontaneous emission. Phys. Rev. Lett. 59: 1189-1192.

69. Frolov S V, Vardeny Z V and Yoshino K, 1998. Cooperative and stimulated emission in poly(p-phenylene-vinylene) thin films and solutions. Phys. Rev. B. 57: 9141-9147.

70. Genack A Z and Drake J M, 1994. Scattering for super-radiance. Nature. 368: 400-401.

71. Zhang Zh-Q, 1995. Light amplification and localization in randomly layered media with gain. Phys. Rev. B. 52, 7960-7964.

72. Wiersma D S and Lagendijk Ad, 1996. Light diffusion with gain and random lasers. Phys. Rev. B. 54: 4256-4265.

73. John S and Pang G, 1996. Theory of lasing in a multiple-scattering medium. Phys. Rev. A. 54: 3642-3652.

74. Thouless D J, 1977. Maximum metallic resistance in thin wires. Phys. Rev. Lett. 18: 11671169.

75. Genack A Z, 1990. Universality of wave propagation in random media. Europhys. Lett. 11: 733-738.

76. Ahmed S A, Zang Zh-W, Yoo K M, Ali M A and Alfano R R, 1994. Effect of multiple light scattering and self-absorption on the fluorescence and excitation spectra of dyes in random media. Appl. Opt. 33: 2746-2750.

77. Frolov S V, Vardeny Z V, Yoshino K, Zakhidov A and Baughman R H, 1999. Stimulated emission in high-gain organic media. Phys. Rev. B. 59: R5284-R5287.

78. Apalkov V M, Raikh M E and Shapiro B, 2002. Random resonators and prelocalized modes in disordered dielectric films. Phys. Rev. Lett. 89: 016802-4.

79. Mujumdar S, Ricci M, Torre R and Wiersma D S, 2004. Amplified extended modes in random lasers. Phys. Rev. Lett. 93: 053903-4.

80. Pinheiro F A and Sampaio L C, 2006. Lasing threshold of diffusive random lasers in three dimensions. Phys. Rev. A. 73: 013826-4.

81. Cao H, 2003. Lasing in random media. Waves in Random Media. 13: R1-R39.

82. Tessler N, Denton G J and Friend R H, 1996. Lasing from conjugated-polymer microcavities. Nature. 382: 695-697.

83. Hide F, Diaz-Garcia M A, Schwartz B J, Andersson M R, Pei Q and Heeger A J, 1996. Semiconducting polymers: A new class of solid-state laser materials. Science. 273: 1833-1836.

84. Brouwer H J, Krasnikov V I, Hilberer A and Hadziioannou G, 1996. Blue superradiance from neat semiconducting alternating copolymer films. Adv. Mater. 8: 935-937.

85. Gelinck G H, Warman J M, Remmers M and Neher D, 1997. Narrow-band emissions from 
conjugated-polymer films. Chem. Phys. Lett. 265: 320-326.

86. Frolov S V, Gellermann W, Ozaki M, Yoshino K and Vardeny Z V, 1997. Cooperative emission in $\pi$-conjugated polymer thin films. Phys. Rev. Lett. 78: 729-732.

87. Long X, Malinowski A, Bradley D D C, Inbasekaran M and Woo E P, 1997. Emission processes in conjugated polymer solutions and thin films. Chem. Phys. Lett. 265: 320-326.

88. Gouedard C, Husson D, Sauteret C, 1993. Generation of spatially incoherent short pulses in laser-pumped neodymium stoichiometric crystals and powders. J. Opt. Soc. Am. B. 10: 23582363.

89. Williams G R, Bayram S B, Rand S C, Hinklin T and Laine R M, 2002. Laser action in strongly scattering rare-earth-metal-doped dielectric nanophosphors. Phys. Rev. A. 65: 013807-6.

90. Zhang D, Cheng B, Yang J, Zhang Y, Hu W and Li Zh, 1995. Narrow-bandwidth emission from a suspension of dye and scatterers. Opt. Commun. 118: 462-465.

91. Sha W L, Liu C-H and Alfano R R, 1994. Spectral and temporal measurements of laser action of Rhodamine 640 dye in strongly scattering media. Opt. Lett. 19: 1922-1924.

92. Balachandran R M and Lawandy N M, 1995. Interface reflection effects in photonic paint. Opt. Lett. 20: 1271-1273.

93. Zhang D, Cheng B, Yang Ju, Zhang Yu, Hu W and Li Zh, 1995. Narrow-bandwidth emission from a suspension of dye and scatterers. Opt. Commun. 118: 462-465.

94. Wiersma D S, van Albada M P and Lagendijk Ad, 1995. Random laser? Nature. 373: 203 204.

95. Lavandy N M and Balachandran R M, 1995. Random laser? Reply. Nature. 373: 204.

96. Lavandy N M, Balachandran R M, Gomes A S L and Sauvain E, 1994. Laser action in strongly scattering media. Nature. 368: 436-438.

97. Ter-Garielyan N E, Markushev V M, Belan V R, Briskina Ch M, Dimitrova O V, Zolin V F and Lavrov A V, 1991. Stimulated radiation emitted by lithium neodymium tetraphosphate LiNd (PO3)4 and neodymium pentaphosphate $\mathrm{NdP}_{5} \mathrm{O}_{14}$ powders. Sov. J. Quant. Electron. 21: 840-842.

98. Cao H, Zhao Y G, Ong H C, Ho S T, Dai J Y, Wu J Y and Chang R P H, 1998. Ultraviolet lasing in resonators formed by scattering in semiconductor polycrystalline films. Appl. Phys. Lett. 73: 3656-3658.

99. Cao H, Zhao Y G, Ho S T, Seelig E W, Wang Q H and Chang R P H, 1999. Random laser action in semiconductor powder. Phys. Rev. Lett. 82: 2278-2281.

100. Cao H, Xu J Y, Chang S-H and Ho S T, 2000. Transition from amplified spontaneous emission to laser action in strongly scattering media. Phys. Rev. E. 61: 1985-1989.

101. Cao H, Xu J Y, Zhang D Z, Chang S-H, Ho S T, Seelig E W, Liu X and Chang R P H, 2000. Spatial confinement of laser light in active random media. Phys. Rev. Lett. 84: 5584-5587.

102. Cao H, Ling Y, Xu J Y, Cao C Q and Kumar P, 2001. Photon statistics of random lasers with resonant feedback. Phys. Rev. Lett. 86: 4524-4527.

103.Wiersig J, Unterhinninghofen J, Song Q, Cao H, Hentschel M and Shinohara S, Chapter 4. Review on unidirectional light emission from ultralow-loss modes in deformed microdisks. In: Trends in nano- and micro-cavities. Ed. by O'Dae Kwon and Byoungho Lee (Kyungwon An. Bentham Science Publishers Ltd., 2011) 109-152.

104. Yamilov A and Cao H, Chap. 44. Self-optimization of optical confinement and lasing action in disordered photonic crystals. In: Optical properties of photonic structures: interplay of order and disorder. Ed. by M F Limonov and R De La Rue (Abingdon: Taylor and Francis Group, 2012) 395-414. 
105. Cao H and Noh H, Chap. 9. Lasing in amorphous nanophotonic structures. In: Amorphous nanophotonics. Ed. by C Rockstuhl and T Scharf (Berlin Heidelberg: Springer, 2013) 227265.

106. Cao H, 2005. Review on latest developments in random lasers with coherent feedback. J. Phys. A: Math. Gen. 38: 10497-10535.

107. Sebbah P and Vanneste C, 2002. Random laser in the localized regime. Phys. Rev. B. 66: 144202-10.

108. Smuk A, Lazaro E, Olson L P and Lawandy N M, 2011. Random laser action in bovine semen. Opt. Commun. 284: 1257-1258.

109. Van der Molen K L, Tjerkstra R W, Mosk A P and Lagendijk Ad, 2007. Spatial extent of random laser modes. Phys. Rev. Lett. 98: 143901-4.

110. Türeci H E, Stone A D and Collier B, 2006. Self-consistent multimode lasing theory for complex or random lasing media. Phys. Rev. A. 74: 043822-17.

111.Türeci H E, Stone A D and Ge L, 2007. Theory of the spatial structure of nonlinear lasing modes. Phys. Rev. A. 76: 013813-4.

112.Türeci H E, Ge L, Rotter S and Stone A D, 2008. Strong interactions in multimode random lasers. Science. 320: 643-646.

113. Haken H and Sauermann H, 1963. Nonlinear interaction of laser modes. Z. Phys. 173: 261275.

114.Leonetti M, Conti C and Lopez C, 2001. The mode-locking transition of random lasers. Nature Photonics. 2: 615-617.

115. Bergman D J and Stockman M I, 2003. Surface plasmon amplification by stimulated emission of radiation: Quantum generation of coherent surface plasmons in nanosystems. Phys. Rev. Lett. 90: 027402-4.

116. Stockman M I, 2011. Nanoplasmonics: past, present, and glimpse into future. Opt. Express. 19: 22029-22106.

117.Stockman M I, 2010. The spaser as a nanoscale quantum generator and ultrafast amplifier. J. Opt. 12: 024004-13.

118. Noginov M A, Zhu G., Belgrave A M, Bakker R, Shalaev V M, Narimanov E E, Stout S, Herz E, Suteewong T and Wiesner U, 2009. Demonstration of a spaser-based nanolaser. Nature. 460: $1110-1113$.

119. Stockman M I, 2008. Spasers explained. Nature Photonics. 2: 327-329.

120.Zheludev N, Prosvirnin S, Papasimakis N and Fedotov V, 2008. Lasing spaser. Nature Photonics. 2: 351-354.

121.Redding B, Choma M A and Cao H, 2012. Speckle-free laser imaging using random laser illumination. Nature Photonics. 6: 355-359.

122.Uchida A, Amano K, Inoue M, Hirano K, Naito S, Someya H, Oowada I, Kurashige T, Shiki M, Yoshimori S, Yoshimura K and Davis P, 2008. Fast physical random bit generation with chaotic semiconductor lasers. Nature Photonics. 2: 728-732.

123.http://phys.org/news148660964.html

124.http://www.sciencedaily.com/releases/2008/05/080523074709.htm

125. Cao H, 2005. Random lasers: Development, features and applications. Optics and Photonics News. 16: 24-29.

126.Polson R C and Vardeny Z V, 2004. Random lasing in human tissues. Appl. Phys. Lett. 85: $1289-1291$.

127.Jeong K-Y, Lee Y-H, Cao H and Yang J-K, 2012. Lasing in localized mode at optimized photonic amorphous structure. Appl. Phys. Lett. 101: 091101-4. 
128. Simonis P and Berthier S, 2012. How nature produces blue color. In: Photonic Crystals - Introduction. Ed. by A. Massaro, Applications and Theory. (Rijeka: InTech). 3-24.

129. Saranathan V, Forster J D, Noh H, Liew S-F, Mochrie S G J, Cao H, Dufresne E R and Prum R O, 2012. Structure and optical function of amorphous photonic nanostructures from avian feather barbs: a comparative small angle X-ray scattering (SAXS) analysis of 230 bird species. J. R. Soc. Interface. 9: 2563-2580.

130. Yang J-K, Boriskina S V, Noh H, Rooks M J, Solomon G S, Negro L D and Cao H, 2010. Demonstration of laser action in a pseudorandom medium. Appl. Phys. Lett. 97: 223101-3.

131. Mahler L, Tredicucci A, Beltram F, Walther Ch, Faist J, Beere H E, Ritchie D A and Wiersma D S, 2010. Quasi-periodic distributed feedback laser. Nature Photonics. 4: 365-369.

132. Segev M, Silberberg Ya and Christodoulides D N, 2013. Anderson localization of light. Nature Photonics. 7: 197-204.

Nastishin Yu. A. and Dudok T. H., 2013. Optically pumped mirrorless lasing. A review. Part I. Random lasing. Ukr. J. Phys. Opt. 14: 146-170.

Анотація. На даний час бездзеркальна лазерна генерація з оптичним нагнітанням представлена трьома окремими напрямами, які стосуються лазерної генерації активних середовищ різних типів: оптично випадкових середовищах, фотонних середовищах і мікрорезонаторах. Ця стаття є першою частиною огляду літератури з бездзеркальної лазерної генерації з оптичним нагнітанням. Ї̈ предмет - випадкова лазерна генерація в розсіювальних середовищах. Інші механізми бездзеркальної лазерної генерації буде обговорено в другій частині огляду. Розглядаючи локалізацію світла як ключову функцію зворотного зв'язку, ми з'ясовуємо можливі механізми локалізації світла в розсіювальних середовищах. Особливу увагу надано локалізаџії світла за Андерсоном. Інші механізми локалізації світла в розсіювальних середовищах стосуються високодобротних резонансів в локальних мікрорезонаторах, щзо існують за рахунок неоднорідності розсіювального середовища. Коротко розглянуто застосування випадкових лазерів. 